\title{
Influence of implant cover screw loosening on early peri-implant marginal bone loss: a retrospective evaluation of risk factors
}

\section{Yin Wuwei}

Guangdong Province Stomatological Hospital

\section{Yang Yong}

Guangdong Province Stomatological Hospital

\section{Wu Zhenzhen}

Guangdong Province Stomatological Hospital

\section{Chen Pei}

Guangdong Province Stomatological Hospital

\section{Yang Zinan}

Guangdong Province Stomatological Hospital

\section{Gan Zekun}

Guangdong Province Stomatological Hospital

\section{Zhou Tengfei}

Guangdong Province Stomatological Hospital

\section{Zeng Mianyan}

Guangdong Province Stomatological Hospital

Chu Hongxing

Guangdong Province Stomatological Hospital

Rong Mingdeng ( $\nabla$ rmdeng@smu.edu.cn )

Guangdong Province Stomatological Hospital

\section{Research Article}

Keywords: dental implants, cover screw, marginal bone loss, case-control study

Posted Date: February 24th, 2021

DOI: https://doi.org/10.21203/rs.3.rs-223040/v1

License: (1) (1) This work is licensed under a Creative Commons Attribution 4.0 International License.

Read Full License 


\section{Abstract}

Background: This study aimed to evaluate the risk factors of cover screw loosening (CSL) and its influence on peri-implant marginal bone during early bone healing process.

Methods: A total of 91 implants in 90 patients ( 75 normal patients and 15 patients with CSL) were included in this study. Panoramic radiographs were taken for the measurement of peri-implant marginal bone loss (MBL). A piece of soft tissue above the cover screw during the second-stage surgery was taken for pathological examination. The differences between the two groups were evaluated and the possible risk factors associated with CSL were analyzed.

Results: Increased frequency of CSL showed significant association with maxillary region $(p=0.038)$. The mean MBL at implants with CSL was $0.82 \pm 0.56 \mathrm{~mm}$, and it was $0.22 \pm 0.20 \mathrm{~mm}$ at normal implants $(p<0.001)$. The inflammatory granulation tissue around the loose cover screws showed infiltration of a large number of inflammatory cells.

Conclusions: CSL during healing process is associated with early peri-implant MBL. The location of the maxilla is considered as a significant risk factor for CSL.

\section{Background}

Crestal bone stability around implants is essential for the long-term success of the dental implant treatment. Generally, a marginal bone loss (MBL) of less than $1.5 \mathrm{~mm}$ in the first year after functional loading and less than $0.2 \mathrm{~mm}$ annually thereafter has been accepted as successful in implant dentistry. ${ }^{(1)}$ Progressive peri-implant MBL promotes colonization of bacteria and induces development of periimplantitis, leading to loss of implant finally. (2) Therefore, preservation of crestal bone and minimization of peri-implant MBL should be considered at the time of implant placement by the dentists.

Several studies have discussed the factors related to peri-implant MBL, including but not limited to some implant design characteristics, the type of implant-abutment connection ${ }^{(3)}$, abutment-height and connection timing ${ }^{(4)}$, number of surgical stages ${ }^{(5)}$, soft tissue thickness ${ }^{(6)}$, microgap leakage ${ }^{(7)}$, overloading $^{(8)}$ and some other host-related factors such as smoking, plaque control, etc. One of the most commonly accepted theory with regard to peri-implant $\mathrm{MBL}$ is that microgap leakage occurs due to microbial leakage, and this is because bacterial infection is the initiator of peri-implantitis and periimplant mucositis. Non-submerged implants are exposed to oral environment partially during bone healing, and are thus considered to be more vulnerable to infection when compared to the submerged ones. Actually, early MBL of non-submerged implants showed association with salivary microbiome. ${ }^{(10)}$

If the primary stability of the implant is less than $10 \mathrm{~N} \cdot \mathrm{mm}$ or if there is any bone deficiency that requires ridge augmentation procedures, then the healing of submerged implant is usually regarded as a better choice to avoid microbial contamination and preserve peri-implant crestal bone. However, early exposure of cover screw accelerates peri-implant MBL, which results in the breakdown of mucosal seal during bone 
healing. ${ }^{(11)}$ Interestingly, patients are occasionally encountered with cover screw loosening (CSL) at the second-stage surgery and found concaved bone resorption around the implant platform. Whether CSL is associated with peri-implant MBL during submerged healing process has not yet been reported.

Hence, this study aimed to evaluate the possible risk factors of CSL and its influence on the peri-implant MBL during early bone healing process. This study might provide insights on our understanding of these topics and enlighten us with regard to reduction and avertion of screw-related complications.

\section{Methods}

\section{Study design and patients recruitment}

The patients who received primarily submerged two-piece microrough titanium dental implant placement and who were scheduled for a regular second-stage surgery at the Department of Periodontology and Implantology, Stomatological Hospital of Southern Medical University from January 2017 to January 2019 were enrolled. The inclusion criteria were as follows: Chinese adult patients who have good general health, non-smokers, underwent positioning of submerged implant in the posterior region, with no history of periodontitis, osteoporosis or regularly take medications that affect the bone metabolism. The exclusion criteria were as follows: patients with immediate or early implant placement, requirement for guided bone regeneration or other augmentation procedures, and mobility of adjacent teeth was greater than 1 degree. All the data and information come from previous medical records, photographs, radiographs and pathological examinations.

\section{Clinical procedures}

All patients underwent two-stage surgical implantation therapy during the study period. The first surgery was performed 3 months after tooth extraction. Implants were placed according to the manufacturer's instruction, and cover screws were tightened manually with a torque of 4-5 N.cm. Implants were placed $0-1 \mathrm{~mm}$ below the bone level, and no bone augmentation procedure was performed. Amoxicillin was given to all patients for three days. Mouth rinsing with $0.12 \%$ chlorhexidine three times a day for a week was prescribed. Patients were instructed to avoid brushing the surgical sites before the sutures were removed.

The second surgery was performed after 3 months in the mandible and 4 months in the maxilla. The thickness of the gingiva was measured in photos recording a periodontal probe and the gingiva after flapping. If a cover screw could be easily loosened with no resistance, then it is defined as CSL. A small piece of connective tissue around/above the cover screw was taken for biopsy and then fixed in $4 \%$ paraformaldehyde before dispatching to the laboratory for histological analysis. Only when there was granulation tissue around the implant, or when there was too much soft tissue that needs to be removed for better suturing, part of the tissue would be cut for biopsy and informed consent of those patients were obtained.

\section{Radiographic measurement}


Panoramic radiography (Sirona ORTHOPHOS XG PLUS) was performed to examine patients immediately after implant placement and on the day of healing cap insertion. The vertical distance from the mesial and distal points of the implant platform to the crestal bone was measured by an independent investigator using radiographic software (Carestream Vue PACS). The distance was represented with positive (+) and negative (-) values according to the location of marginal bone coronally (+) or apically (-) to the implant platform, and the mean value of each implant was obtained. MBL was measured by subtracting the obtained data at two time points. All these evaluations were performed twice with a time interval of 2 weeks.

\section{Statistical analysis}

All statistical analyses were performed using SPSS Statistics 25 (IBM, USA). The factors associated with CSL were determined by Pearson's chi-square test and cross-table analysis. The mean values and ranges of MBL were calculated for the two groups. The normality of distribution was tested with KolmogorovSmirnov test. Data analysis was performed by Student's $t$ test when the distribution was normal. A $P$ value of $<0.05$ was considered to be statistically significant.

\section{Results}

Ninety patients ( 61 females and 29 males, aged 20 to 73 years) who have received 91 implants from January 2017 to January 2019 were included in the analysis. 15 implants in 15 patients revealed CSL during second surgery, and the remaining 76 implants were found to be normal. No significant differences in age and gender were discovered between the two groups (Table 1). Statistical analysis revealed increased frequency of CSL with implants placed in the maxillary region when compared to those placed in the mandibular region $(p=0.038)$. No significant association was observed between the frequency of CSL and vertical mucosa thickness $(p=0.723)$ as well as the implant designs including the connection type $(p=0.167)$, height $(p=0.939)$ and diameter $(p=0.355)$ of cover screws (Table 2$)$.

The mean MBL of the implants with CSL was $0.82 \pm 0.56 \mathrm{~mm}$, while that of normal implants was $0.22 \pm$ $0.20 \mathrm{~mm}$ (Table 3). The Kolmogorov-Smirnov test showed that the data is normally distributed. The two groups showed statistically significant differences in MBL (Student-t test, $P<0.001$ ). Granulation tissue infiltration with numerous inflammatory cells was observed in the bone defect area around the implants with CSL, while implants without CSL were covered with healthy gingival tissue (Fig. 1). 
Table 1

Demographic parameters of included patients.

\begin{tabular}{|llll|}
\hline Parameters & Without CSL & Revealing CSL & $\boldsymbol{p}$ \\
\hline Age (years) & & & \\
\hline Mean (SD) & $40.2(14.0)$ & $43.0(12.4)$ & \\
\hline Range & $20-73$ & $23-64$ \\
\hline Median & 37.0 & 44.0 \\
\hline Agegroups & & & \\
\hline $18-25$ & $13(17.3 \%)$ & $1(6.7 \%)$ & \\
\hline $26-35$ & $20(26.7 \%)$ & $5(33.3 \%)$ & \\
\hline $36-49$ & $24(32.0 \%)$ & $3(20.0 \%)$ & \\
\hline $50-59$ & $10(13.3 \%)$ & $5(33.3 \%)$ & 0.399 \\
\hline $60-69$ & $6(8.0 \%)$ & $1(6.67 \%)$ & \\
\hline$\geq 70$ & $2(2.7 \%)$ & $0(0.0 \%)$ & 0.614 \\
\hline Total & 75 & 15 \\
\hline Gender & & & \\
\hline Female & $50(66.7 \%)$ & $11(60.0 \%)$ & \\
\hline Male & $25(33.3 \%)$ & $4(40.0 \%)$ & \\
\hline
\end{tabular}


Table 2

Assessment of factors associated with cover screw loosening at implant level.

\section{Parameters \\ $p \quad$ Remarks}

Maxilla/mandible

0.038

Without

CSL

Revealing

CSL

Maxillary sites

$20(26.3 \%) \quad 8(50.0 \%)$

Mandibular sites

$56(73.7 \%) \quad 7(50.0 \%)$

Implant system

0.482

SICace $^{*}$

Astra Tech ${ }^{\dagger}$

$\operatorname{ICX} \ddagger$

Zimmer T3§
0.318

Conical connection

Without conical connection

0.355

$1.5 \mathrm{~mm}$

$1.75 \mathrm{~mm}$

$2.0 \mathrm{~mm}$

0.939

Higher than the implant platform

Flush with the implant platform

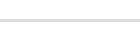

$36(47.4 \%) \quad 5(33.3 \%)$

$19(25.0 \%) \quad 6(40.0 \%)$

$17(28.3 \%) \quad 4(26.7 \%)$

$4(5.3 \%)$

$0(0.0 \%)$

Height of cover screw

Vertical mucosal

thickness

0.369

40 (52.6\%) 10 (66.7\%)

$36(47.4 \%) \quad 5(33.3 \%)$

$40(52.6 \%) \quad 5(33.3 \%)$

$17(22.4 \%) \quad 4(26.7 \%)$

$19(25.0 \%) \quad 6(40.0 \%)$

$55(72.4 \%) \quad 11(73.3 \%)$

$21(27.6 \%) \quad 4(26.7 \%)$

* SICace implants (SIC invent, Switzerland)

† Astra Tech implants (Dentsply, Sweden)

‡ ICX implants (medentis medical GmbH, Germany)

§ Zimmer T3 implants (Zimmer Biomet, USA). 


\begin{tabular}{|c|c|c|c|c|}
\hline Parameters & $p$ & Remarks & & \\
\hline & & $>2 \mathrm{~mm}$ & $27(35.5 \%)$ & $3(20.0 \%)$ \\
\hline & & $\leq 2 \mathrm{~mm}$ & $49(64.5 \%)$ & $12(80.0 \%)$ \\
\hline \multicolumn{5}{|c|}{ * SICace implants (SIC invent, Switzerland) } \\
\hline \multicolumn{5}{|c|}{ † Astra Tech implants (Dentsply, Sweden) } \\
\hline \multicolumn{5}{|c|}{ ‡ ICX implants (medentis medical GmbH, Germany) } \\
\hline$\S$ Zimmer T3 & & net, USA). & & \\
\hline
\end{tabular}

Table 3

Peri-implant marginal bone loss

\begin{tabular}{|c|c|c|c|}
\hline Marginal bone loss (mm) & Revealing CSL & Without CSL & $p$ \\
\hline Mean (SD) & $0.82(0.56)$ & $0.22(0.20)$ & $\varangle 0.001$ \\
\hline Range & $0.08-1.88$ & $-0.15-0.86$ & \\
\hline Median & 0.83 & 0.20 & \\
\hline
\end{tabular}

\section{Discussion}

Nowadays, two-piece implants with internal connection occupy a large space in the market of dental implant systems. Cover screw was designed to prevent growing of hard and soft tissues into the intraimplant cavity during bone healing process. The implant systems have different internal morphologies, and the cover screws differ from one another in shape and diameter. The primary objective of this study was to identify the possible risk factors of CSL, assuming that the implant site (maxilla/mandible), the thickness of gingiva and different implant designs might be associated with increased frequency of CSL. The results in the present study showed that the implants in the posterior region of the maxilla are more prone to CSL than those present in the posterior region of the mandible $(p=0.038)$. The location of the maxilla was shown to be a significant risk factor for early failure of dental implants ${ }^{(12)}$, and more periimplant MBL was observed in the maxilla. ${ }^{(13)}$ Theoretically, cover screws higher than the implant platform are more susceptible to external forces. The diameter of a larger screw occupies increased area between the screw and the fixture, thus providing greater frictional retention force. ${ }^{(14)}$ Also conical connection that is originated from the concept of Morse taper ensures a more reliable retention. ${ }^{(15)}$ However, none of the factors related to cover screw configurations presented in this study showed association with higher 
frequency of CSL. It is more like a random low-probability event that has nothing to do with the design of the implant system.

But how could a cover screw become loose by itself under the cover of the gums? And this might be perhaps due to not completely tightened from the beginning because of the clinician's improper operations. For example, the implant body might rotate along with the cover screw when the primary stability remains poor due to over-preparation of implant bed ${ }^{(16)}$, so that the tightening torque is not enough to hold the screw joint together. The cover screw cannot be fully seated, and small particles such as bone graft materials get struck in the way accidentally, or it is inserted in a wrong direction, so that the threads are mismatched. Once the CSL occurs, then the peri-implant marginal bone resorption is greatly increased. Our results showed a statistically significant difference in MBL between the implants with CSL and normal implants $(P<0.001)$. Implants with CSL had a more severe MBL of $0.82 \mathrm{~mm}$ in just 3-4 months, which is a sign of worse prognosis from a clinical point of view. Physiological bone remodeling within the first year after implant placement is an adaptive process to surgical trauma and biological width establishment. ${ }^{(17)}$ The etiologies of early MBL around dental implants have not been well understood yet. The risk factors associated with MBL could be mainly divided into mechanical factors and biological factors. ${ }^{(18)}$

In this study, increased early MBL related to CSL during bone healing of the canals can be explained from these two aspects. A microgap between implant and screw threads allows the passage of fluids containing bacteria and metabolic products to the implant cavity. With the presence of implant-screw microgap, additional micromovement under external forces causes thread slippage, eventually leading to screw loosening. ${ }^{(19,20)}$ Despite strict preoperative disinfection, oral cavity is still regarded as a bacterial environment. Bacterial contamination can be found in the intra-implant cavity both in the implants with healthy and diseased tissue conditions. ${ }^{(21)}$ The microbial leakage that is associated with micromotion in two-piece implants might have a connection with significant inflammatory cell infiltration, leading to MBL. ${ }^{(22,23)}$ Simultaneously, the titanium debris and the particles that are discharged from the mechanical wear of implant-screw interface could leak out through the microgap and activate the immune responses, showing association of biomedical mediators with bone resorption. ${ }^{(24)}$ Fibrous tissue encapsulation contributes to excessive micromotion ${ }^{(25)}$, and might produce a combined effect on destruction of osseointegration. The granulation tissue formation was observed between the moving implants and new bone in a mouse model of implant micromotion system. ${ }^{(26)}$ The presence of granulation tissue around the loose cover screws in our study is consistent with the results of their study.

The main limitations of this study were the inclusion of small number of patients. Many patients with preexisting bone deficiency, periodontitis and smoking habits were excluded in order to eliminate other factors that affect the peri-implant MBL. Coupled with low incidence of CSL, only 90 patients were included in the two years. Also the retrospective study design makes it impossible to supplement some data and details that were not recorded. It is worth mentioning that the marginal bone resorption is a very complex process that is influenced by some host-related factors. Unfortunately, the bias caused by 
individual differences cannot be completely excluded. To further confirm the surgeon-related factors associated with CSL, a prospective clinical trial should be conducted.

\section{Conclusions}

This study aimed to evaluate the influence of CSL on early peri-implant MBL. CSL during submerged healing process is associated with peri-implant MBL. The location of the maxilla is considered as a significant risk factor for CSL. Improper operations during implant placement that might increase the risk of CSL should be avoided as much as possible. Further investigations and clinical trials are warranted in order to confirm the causative mechanism of early MBL led by CSL.

\section{Abbreviations}

CSL: Cover screw loosening; MBL: Marginal bone loss;

\section{Declarations}

\section{Consent for publication}

Not applicable.

\section{Ethics approval and consent to participate}

This study was approved by the Ethics Committee of the Stomatological Hospital of Southern Medical University. All methods were carried out in accordance with relevant guidelines and regulations. All subjects were informed of the procedure of this study. The need for signed informed consent forms was waived by the Ethics Committee of the Stomatogolical Hospital of Southern Medical University. Because it's a retrospective study and the research presents no more than minimal risk of harm to subjects.

\section{Availability of data and materials}

The datasets used in current study are available from the corresponding author on reasonable request.

\section{Competing interest}

The authors declare that they have no competing interest.

\section{Funding}

This study was supported by grants from Southern Medical University Stomatological Hospital (PY2017031) and Guangdong Province (2018KJ2014).

\section{Authors' contributions}


Dr. Wuwei Yin and Dr. Yong Yang contributed equally to this article. WY and YY wrote the main manuscript text. RM designed the study. WZ and CP collected the data. YZ, GZ, and ZT did the radiographic measurement. $\mathrm{ZM}$ and $\mathrm{CH}$ did the statistical analysis. All authors read and approved the final manuscript. All authors have read and approved the manuscript.

\section{Acknowledgements}

Not applicable.

\section{References}

1. Misch CE, Perel ML, Wang HL, Sammartino G, Galindo-Moreno P, Trisi P, et al. Implant success, survival, and failure: the International Congress of Oral Implantologists (ICOI) Pisa Consensus Conference. Implant Dent. 2008;17(1):5-15.

2. Galindo-Moreno P, Leon-Cano A, Ortega-Oller I, Monje A, F OV, Catena A. Marginal bone loss as success criterion in implant dentistry: beyond 2 mm. Clin Oral Implants Res. 2015;26(4):e28-e34.

3. Qian J, Wennerberg A, Albrektsson T. Reasons for marginal bone loss around oral implants. Clin Implant Dent Relat Res. 2012;14(6):792-807.

4. Borges T, Leitao B, Pereira M, Carvalho A, Galindo-Moreno P. Influence of the abutment height and connection timing in early peri-implant marginal bone changes: A prospective randomized clinical trial. Clin Oral Implants Res. 2018;29(9):907-14.

5. Gheisari R, Eatemadi H, Alavian A. Comparison of the Marginal Bone Loss in One-stage versus Twostage Implant Surgery. J Dent (Shiraz). 2017;18(4):272-6.

6. Suarez-Lopez Del Amo F, Lin GH, Monje A, Galindo-Moreno P, Wang HL. Influence of Soft Tissue Thickness on Peri-Implant Marginal Bone Loss: A Systematic Review and Meta-Analysis. J Periodontol. 2016;87(6):690-9.

7. Hermann JS, Schoolfield JD, Schenk RK, Buser D, Cochran DL. Influence of the size of the microgap on crestal bone changes around titanium implants. A histometric evaluation of unloaded nonsubmerged implants in the canine mandible. J Periodontol. 2001;72(10):1372-83.

8. Naert I, Duyck J, Vandamme K. Occlusal overload and bone/implant loss. Clin Oral Implants Res. 2012;23 Suppl 6:95-107.

9. Smeets R, Henningsen A, Jung O, Heiland M, Hammacher C, Stein JM. Definition, etiology, prevention and treatment of peri-implantitis-a review. Head Face Med. 2014;10:34.

10. Duan XB, Wu TX, Guo YC, Zhou XD, Lei YL, Xu X, et al. Marginal bone loss around non-submerged implants is associated with salivary microbiome during bone healing. Int J Oral Sci. 2017;9(2):95103.

11. Kim TH, Lee DW, Kim CK, Park KH, Moon IS. Influence of early cover screw exposure on crestal bone loss around implants: intraindividual comparison of bone level at exposed and non-exposed implants. J Periodontol. 2009;80(6):933-9. 
12. Manzano G, Montero J, Martín-Vallejo J, Del Fabbro M, Bravo M, Testori T. Risk Factors in Early Implant Failure: A Meta-Analysis. Implant Dent. 2016;25(2):272-80.

13. Vervaeke S, Collaert B, Cosyn J, Deschepper E, De Bruyn H. A multifactorial analysis to identify predictors of implant failure and peri-implant bone loss. Clin Implant Dent Relat Res. 2015;17 Suppl $1: e 298-307$.

14. Shin HM, Huh JB, Yun MJ, Jeon YC, Chang BM, Jeong CM. Influence of the implant-abutment connection design and diameter on the screw joint stability. J Adv Prosthodont. 2014;6(2):126-32.

15. Schmitt CM, Nogueira-Filho G, Tenenbaum HC, Lai JY, Brito C, Doring H, et al. Performance of conical abutment (Morse Taper) connection implants: a systematic review. J Biomed Mater Res A. 2014;102(2):552-74.

16. Javed F, Ahmed HB, Crespi R, Romanos GE. Role of primary stability for successful osseointegration of dental implants: Factors of influence and evaluation. Interv Med Appl Sci. 2013;5(4):162-7.

17. Lombardi T, Berton F, Salgarello S, Barbalonga E, Rapani A, Piovesana F, et al. Factors Influencing Early Marginal Bone Loss around Dental Implants Positioned Subcrestally: A Multicenter Prospective Clinical Study. J Clin Med. 2019;8(8).

18. Insua A, Monje A, Wang HL, Miron RJ. Basis of bone metabolism around dental implants during osseointegration and peri-implant bone loss. J Biomed Mater Res A. 2017;105(7):2075-89.

19. Michalakis KX, Calvani PL, Muftu S, Pissiotis A, Hirayama H. The effect of different implantabutment connections on screw joint stability. J Oral Implantol. 2014;40(2):146-52.

20. Winkler S, Ring K, Ring JD, Boberick KG. Implant Screw Mechanics and the Settling Effect: An Overview. Journal of Oral Implantology. 2003;29(5):242-5.

21. Canullo L, Penarrocha-Oltra D, Covani U, Rossetti PH. Microbiologic and Clinical Findings of Implants in Healthy Condition and with Peri-Implantitis. Int J Oral Maxillofac Implants. 2015;30(4):834-42.

22. Jervoe-Storm PM, Jepsen S, Johren P, Mericske-Stern R, Enkling N. Internal bacterial colonization of implants: association with peri-implant bone loss. Clin Oral Implants Res. 2015;26(8):957-63.

23. Liu Y, Wang J. Influences of microgap and micromotion of implant-abutment interface on marginal bone loss around implant neck. Arch Oral Biol. 2017;83:153-60.

24. Apaza-Bedoya K, Tarce M, Benfatti CAM, Henriques B, Mathew MT, Teughels W, et al. Synergistic interactions between corrosion and wear at titanium-based dental implant connections: A scoping review. J Periodontal Res. 2017;52(6):946-54.

25. Szmukler-Moncler S, Salama H, Reingewirtz Y, Dubruille JH. Timing of loading and effect of micromotion on bone-dental implant interface: review of experimental literature. J Biomed Mater Res. 1998;43(2):192-203.

26. Wazen RM, Currey JA, Guo H, Brunski JB, Helms JA, Nanci A. Micromotion-induced strain fields influence early stages of repair at bone-implant interfaces. Acta Biomater. 2013;9(5):6663-74.

\section{Figures}



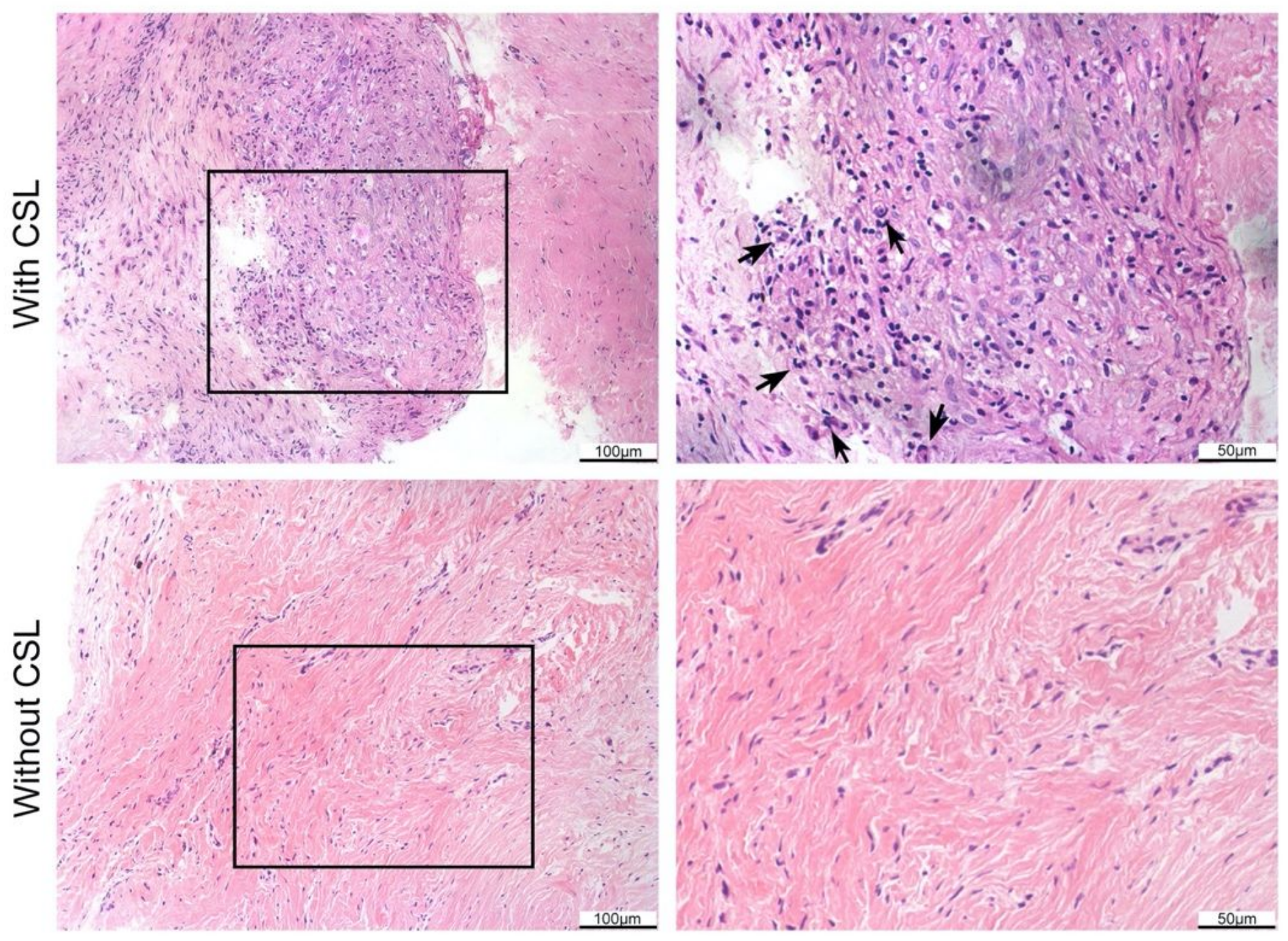

Figure 1

Histological analysis of peri-implant soft tissue. Inflammatory granulation around loose cover screws was infiltrated with inflammatory cells (arrows). 